\title{
The future of psychiatric rehabilitation
}

\author{
R. E. Drake
}

First published online 15 December 2016

Psychiatric rehabilitation helps people with serious mental disorders to improve their functional adjustment and social inclusion. Over the past few decades, psychiatric rehabilitation has developed an extensive evidence base showing that people with even the most severe disorders can attain meaningful functional goals: independent living, competitive employment, satisfying relationships and better quality of life. We now call these changes 'recovery'.

A significant percentage of people in every society experience serious functional difficulties due to mental disorders. Psychiatric rehabilitation could help most of these people to live more independent, satisfying, functional lives. The central problem, however, is lack of access to high-quality services, even in the wealthiest countries. In the USA, for example, fewer than $5 \%$ of people with serious psychiatric disorders are able to access evidence-based psychiatric rehabilitation.

This special section of Epidemiology and Psychiatric Science contains three editorials that discuss the state of psychiatric rehabilitation in Europe, the USA and Latin America, respectively. First, Rossler and Drake advocate a common approach for the convergence of disparate systems across Europe: "Services must be driven by values, client preferences, outcomes research, and implementation science. Common approaches could promote recovery, social inclusion, and cost savings." Second, Bond and Drake describe the paradox of high-quality research and poor-quality implementation in the U.S.: "The U.S. health care system is expensive, inefficient, and diverse - essentially a non-system producing enormous disparities in care." Finally, UribeRestrepo, Escobar, and Cubillos describe the emergence of psychiatric rehabilitation in Latin America: "Evidence-based models of rehabilitation, such as assertive community treatment, family psycho-education, supported housing, and supported employment, should build on current services, resources, and legislation, with adaptations to local conditions and cultures." Europe and the USA exemplify high-income countries with different health care systems. Latin America includes many middle-income countries, where the

* Address for correspondence: R. E. Drake, Dartmouth College and Westat Corporation, Lebanon, New Hampshire, USA.

(Email: Robert.E.Drake@dartmouth.edu) issues are quite different, with a focus on developing new service systems. Meanwhile, psychiatric rehabilitation is almost completely absent in low-income countries.

\section{Global mental health}

In high-income countries, serious psychiatric disorders such as mood and psychotic disorders have already become the leading cause of disability (Organisation for Economic Co-operation and Development). Other countries may soon experience a similar reality. As they join the global economy and increase their per capita gross national products, they are likely to overcome health problems related to infectious disease, civil war, poor sanitation, food insecurity and extreme poverty. Concurrently, chronic, non-infectious diseases - among them, psychiatric disorders are prominent - will become the dominant health problems. In addition to human suffering, psychiatric disability constitutes a serious societal burden. Because mental disorders typically begin in adolescence or early adulthood, they interfere with education and job development, diminish human capital and undermine economic growth.

The policy issues in relation to psychiatric rehabilitation differ substantially across high-income, middleincome and low-income countries. In high-income countries, as the following editorials on Europe and the USA attest, empirical knowledge is not the problem instead, powerful vested interests maintain outmoded systems and obstruct change, with the result that evidence-based practices are generally unavailable. Because people with mental disorders have little wealth and political power, their interests do not garner political attention. Indeed, they are such a disenfranchised and stigmatised group that mental health services are typically overlooked, devalued and diminished during economic downturns.

Hospitals, private mental health treatment organisations, pharmaceutical companies, device manufacturers and psychiatrists do not make money by providing evidence-based psychiatric rehabilitation. In the USA over the past decades, public spending increased for non-evidence-based pharmaceutical practices, while psychiatric rehabilitation programs lost funding. The net result has been that many people with serious mental disorders received expensive 
polypharmacy, even becoming addicted to new medications, and very few have access to rehabilitation. This trend has been antithetical to the expressed interests of people with serious mental disorders.

The policy recommendations in high-income countries have been legion: for examples, implementing accountable care organisations and capitated payments, restructuring payment models and training programs to support integration of services, involving clients and families in the design and implementation of care systems and developing learning communities for all stakeholders. But few organizations (besides the Department of Justice) are able to counter the major offenders.

In middle-income countries, the policy issues are different. As the following editorial on Latin America points out, middle-income countries in Latin America are beginning to focus on the mental health infrastructures that will be needed to address psychiatric rehabilitation, largely by starting with legislative and policy initiatives on basic human rights to health, equality and employment. These policies have influenced service development in a variety of ways, but evidence-based psychiatric rehabilitation has not yet been prioritised and adopted in many areas. Many of these initiatives have instead continued hospital-based or facility-based care rather than community-based interventions.

With its emerging economic resources, Latin America could avoid many of the historical missteps that currently hamper reform in high-income countries, make use of its unique cultural assets and community strengths, and perhaps take advantage of the revolution in health information technology to develop mental health systems that are more functional, person-centred and cost-effective than those in wealthy countries. Several clear barriers include funding, vested interests and politics.

Meanwhile, low-income countries are struggling to enter the global economy, conquer long-standing causes of morbidity and mortality, and develop rudimentary mental health systems. They have opportunities to plan and create psychiatric rehabilitation programs that fit their individual histories, cultures and resources, but they also face numerous risks. One is that former colonial countries will again exploit them. This happens already: wealthy countries that fund their own expensive, flawed mental health systems have sometimes advised poor countries to develop similar professionalised, institution-based mental health systems. Very poor countries have spent scarce resources to develop medical schools and train psychiatrists who then go to wealthy countries for training and never return. The movement to expand evidence-based mental health services in low-income countries does acknowledge that services have not been validated in these countries and must involve lay health workers, but minimal research has addressed local beliefs, customs and cultures. The danger is that leaders in low-income countries will overlook the wisdom of their own people. To develop person-centred care, people with mental illness and their families should be involved in co-creating mental health services from the ground up. They are familiar with local beliefs and resources; they know the most acute needs; they can choose next steps; and they can implement and help to measure the effectiveness of new services. Yet stigma and outside forces may diminish their influence.

\section{Future of psychiatric rehabilitation}

Psychiatric rehabilitation addresses the outcomes most valued by people with serious mental illness: functional status and quality of life. Evidence-based, person-centred practices are available but rarely used and not yet adapted for the variety of countries around the world. Furthermore, the implementation challenges vary across countries, in relation to wealth, location, culture and many other factors. Nevertheless, health care organisations and governments in all countries should have the common goal of helping people with the most severe illnesses to have opportunities to live, work, socialise and participate fully in their communities. To do so, countries must strive to implement evidence-based, person-centred, culturally competent psychiatric rehabilitation.

\section{Acknowledgements}

None.

\section{Financial support}

This research received no specific grant from any funding agency, commercial or not-for-profit sectors.

\section{Conflict of Interest}

None.

\section{References}

Bond G.R., Drake R.E. (2017). New directions for psychiatric rehabilitation in the U.S. Epidemiology and Psychiatric Services, this issue.

Rossler W., Drake R.E. (2017). Psychiatric rehabilitation in Europe. Epidemiology and Psychiatric Services, this issue.

Uribe-Restropo J.M., Escobar M.L., Cubillos L. (2017). Psychiatric rehabilitation in Latin America. Epidemiology and Psychiatric Services, this issue. 